\title{
Approximation of slow attracting manifolds in chemical kinetics by trajectory-based optimization approaches
}

\author{
V. Reinhardt, M. Winckler, and D. Lebiedz* \\ Interdisciplinary Center for Scientific Computing, \\ Im Neuenheimer Feld 368, D-69120 Heidelberg, Germany
}

(Dated: March 20, 2007)

\begin{abstract}
Many common kinetic model reduction approaches are explicitly based on inherent multiple time scales and often assume and directly exploit a clear time scale separation into fast and slow reaction processes. They approximate the system dynamics with a dimension-reduced model after eliminating the fast modes by enslaving them to the slow ones. The corresponding restrictive assumption of full relaxation of fast modes often renders the resulting approximation of slow attracting manifolds inaccurate as a representation of the reduced model and makes the numerical solution of the nonlinear "reduction equations" particularly difficult in many cases where the gap in intrinsic time scales is not large enough. We demonstrate that trajectory optimization approaches can avoid such severe restrictions by computing numerical solutions that correspond to "maximally relaxed" dynamical modes in a suitable sense. We present a framework of trajectory-based optimization for model reduction in chemical kinetics and a general class of reduction criteria characterizing the relaxation of chemical forces along reaction trajectories. These criteria can be motivated geometrically exploiting ideas from differential geometry and fundamental physics and turn out to be highly successful in example applications. Within this framework, we provide results for the computational approximation of slow attracting low-dimensional manifolds in terms of families of optimal trajectories for a six-component hydrogen combustion mechanism.
\end{abstract}

\section{INTRODUCTION}

The idea of modeling chemical kinetics is to map reality to a mathematical description of the system, i.e. to describe its dynamics by differential equations. While reducing reality to equations one has to bear in mind that it is conceptually impossible to describe every microscopic detail. Depending on the task at hand the appropriate degree of accuracy of the system description has to be chosen. For example in a simulation of technical combustion processes not every single molecule has to be tracked on a microscopic level. Instead, usually only a few characteristic "macroscopic" variables are used to describe the phenomenological system behavior of interest.

The art of modeling often consists in finding an appropriate level of abstraction for describing the aspects which are to be investigated. There are two general approaches to model multi-scale problems. One of them is the so called top-down approach. First a rough model of the system phenomena is set up which then is refined according to experimental observations. In the other approach, called bottom-up approach, a system is modeled using first-principle physical and chemical laws, taking into account as much detailed knowledge as available. The latter approach is more precise and describes a system more accurately than the first one. However, it generally leads to large-scale models with huge numbers of variables. For efficient spatiotemporal simulations these high-dimensional models are inappropriate, in particular if they involve multiple time scales causing severe stiff-

*Electronic address: lebiedz@iwr.uni-heidelberg.de ness.

This is where model reduction comes into application. A central issue of model reduction is to address the discrepancy between the need to develop detailed highdimensional multi-scale models (e.g. in chemical kinetics) and the inefficiency of their use in computationally demanding numerical simulations. The ultimate goal of all model reduction techniques in chemical kinetics is to find a low-dimensional approximation of a reaction mechanism which contains all the essential information to still describe the system accurately enough. For a kinetic model set up by the bottom-up approach this is equivalent to identifying the essential degrees of freedom with respect to the system properties of interest. Those properties of interest are often related to long-term dynamics. To construct low-dimensional approximations, many model reduction techniques therefore make use of intrinsic multiple time scales. If the long-term behavior of a system is to be studied, fast transient dynamical modes are assumed to be relaxed within the reduced model approximation replacing the original system of differential equations by one of lower dimension without losing too much key information about the long-term system dynamics.

However, in simulations of technical processes usually all species are relevant for the properties of interest and therefore have to be considered, not only the ones included in the reduced mechanism. Hence the concentrations for the species of the full mechanism need to be automatically calculated as functions of the species of the reduced mechanism. This so-called automatic species reconstruction is implemented in most of the model reduction algorithms, independently of the concepts the methods are based on. 
Comprehensive overviews of the most common model reduction techniques and their underlying concepts can be found in ${ }^{1,2}$. Most modern model reduction methods are based on one of the following three general strategies: lumping ${ }^{3}$, sensitivity analysis ${ }^{4-6}$ and time scale analysis. Model reduction techniques based on time scale analysis range from the quasi-steady-state assumption (QSSA) $)^{7,8}$ and the partial equilibrium approximation $(\mathrm{PEA})^{9}$ to modern computational methods as computational singular perturbation (CSP) methods ${ }^{10,11}$, inertial manifold approaches ${ }^{12}$, intrinsic low-dimensional manifolds $(\text { ILDM })^{13-15}$ and ideas from integer optimization ${ }^{16,17}$ to eliminate "unnecessary" species and reactions.

Apart from methods making explicit use of time scale separation, powerful geometrical approaches to simplification of chemical kinetics have been investigated by Fraser and Roussel ${ }^{18-22}$. Fraser's algorithm is based on a fixed point iteration of a functional equation obtained from the underlying system of differential equations within a phase space formalism. A truncated version of this functional equation has recently been introduced in order to accelerate the computation of ILDMs ${ }^{23}$. Other approaches not explicitly based on time scale separation are the rate-controlled constrained-equilibrium (RCCE) method first proposed by Keck and Gillespie ${ }^{24}$ and later further developed by Hamiroune et al. ${ }^{25}$ and the invariant constrained equilibrium edge pre-image curve (ICE-PIC) method recently introduced by Ren et al. ${ }^{26,27}$.

Lebiedz ${ }^{28}$ presented a novel approach to model reduction in chemical kinetics that is based on the optimization of trajectories subject to given constraints. The resulting trajectories are supposed to be maximally relaxed with respect to an optimization criterion chosen to be minimal entropy production rate $\mathrm{in}^{28}$. This approach assures that at least an approximation of slow attracting manifolds that is "as good as possible" is found even in regions, where other model reduction methods as for example the ILDM $^{13}$ requiring a clear time scale separation fail.

Pursuing Lebiedz's optimality concept, here, we present a generalized trajectory-based optimization approach suitable for the accurate computational approximation of slow attracting low-dimensional manifolds and its adaptation and application to realistic kinetic models and higher-dimensional manifolds. In particular, we develop a novel criterion for the desired maximal relaxation of forces along reaction trajectories parameterizing the reduced model. We motivate our criterion on the background of a geometric interpretation of chemical forces.

\section{GENERAL METHODOLOGY}

In this work, the novel model reduction concept for chemical kinetics first proposed by Lebiedz ${ }^{28}$ is further developed and considerably extended. Our conceptual idea is based on finding optimal criteria related to maximal relaxation of chemical forces along phase space trajectories under given constraints. This idea is exploited by formulating optimization problems for the numerical computation of such trajectories and their use as a representation of a reduced model in terms of slow attracting manifolds spanned by these trajectories.

An important practical issue of model reduction in chemical kinetics is the a priori choice of some species as so-called reaction progress variables which serve as representatives of the reduced model in terms of a parameterization. In our context this finds a fully natural realization in terms of initial conditions of trajectories. For fixed initial values of those progress variables both a special trajectory converging towards the equilibrium point in phase space and the a priori unknown initial values of the remaining species (species reconstruction) are calculated at the same time as a solution of the optimization problem. Our species reconstruction procedure can be interpreted as the maximal relaxation of chemical forces or dynamic modes of a chemical system under the constraints of fixed progress variables.

We will provide an improved optimization criterion suitable for an approximation of slow, invariant attracting manifolds and its numerical implementation and present promising results for the reduction of a realistic test case reaction mechanism describing hydrogen combustion. Our criterion unites ideas from and shares a deep connection to both differential geometry concepts related to the curvature of trajectories and slow longterm dynamics enslaving fast modes in a multiple timescale kinetic system. The well-established geometric interpretation of physical force as curvature of manifolds is transfered to the field of dissipative forces in chemical kinetics.

Our trajectory-based optimization approach for model reduction in chemical kinetics can generally be formulated as

$$
\begin{array}{cll}
\min _{c_{k}} & \int_{0}^{T} \Phi(c(t)) \mathrm{d} t & \\
\text { subject to } & \frac{\mathrm{d} c_{k}}{\mathrm{~d} t}=f_{k}(c), & k=1, \ldots, m \\
& c_{k}(0)=c_{k}^{0}, & k \in I_{\text {fixed }} \\
& \left|c_{k}(T)-c_{k}^{\text {eq }}\right| \leq \varepsilon, & k \in I_{\text {fixed }}
\end{array}
$$

and subject to conservation relations. $c_{k}$ are the concentrations of chemical species, $I_{\text {fixed }}$ is the index set that contains the indices of variables with fixed initial values (the reaction progress variables). The system dynamics is described by (1b) and the initial concentrations of the reaction progress variables are fixed in (1c). When approaching the equilibrium point $c^{\mathrm{eq}}$, the system dynamics become infinitely slow. Therefore the equilibrium point is approximated in (1d) within a surrounding of small radius $\varepsilon$ for the reaction progress variables. A priori the end time $T$ is free and is determined within the optimization such that $(1 \mathrm{~d})$ is fulfilled. Alternatively the time $T$ can be fixed such that the final state of the system is very close to the chemical equilibrium point. The objective functional $\Phi(c(t))$ in (1a) describes an optimization 
criterion related to the degree of relaxation of chemical forces. The choice of the objective functional is discussed below.

For the numerical solution of the boundary value problem stated in (1), elaborate mathematical optimization techniques exist. In the example applications presented in the following, the software package MUSCOD-II 29,30 originally developed for solving large scale optimal control problems for nonlinear dynamical systems is used for the numerical solution of problem (1). In MUSCOD-II the direct multiple shooting method ${ }^{29}$ is implemented. By discretizing the state variables, the originally infinite dimensional problem is transformed into a finite dimensional nonlinear programming problem (NLP), which then can be solved by a sequential quadratic programming (SQP) method. The state trajectories are numerically integrated only on small subintervals of the full time horizon, which are initially decoupled, using a BDF-type (backward differentiation formulae) stiff integrator ${ }^{31}$. As a result, for each multiple shooting interval an initial value problem has to be solved instead of just one for the whole time horizon. Although the resulting NLP is much larger than the one resulting from the single shooting approach, the mathematical structure of the multiple shooting discretization can be exploited such that the related optimization problem can be solved with approximately the same effort as in the single shooting approach $^{32}$. However, compared to single shooting, multiple shooting is much more robust. Furthermore even for systems with dynamical instabilities, e.g. strongly diverging state trajectories, the computation of derivative information which is required for die SQP optimization is much more stable and in some cases even possible at all.

Another significant advantage of the multiple shooting approach is that neighboring problems can be initialized very efficiently from the previous optimal trajectory. In the so called initial value embedding strategy ${ }^{33,34}$ formally a linear extrapolation prediction of the previous solution is used to calculate an initial guess for the new solution if the same problem needs to be solved with just slightly modified initial values. The incorporation of a priori information about the optimal solution (trajectory) by setting initial conditions for the state variables at the multiple shooting nodes generally results in highly improved and fast convergence to the new solution which actually makes the solution of parameterized optimization problems very efficient. This strategy can be efficiently exploited in model reduction, since reduced models often need to be computed and tabulated for a whole range of reaction progress variables.

The initial value embedding strategy is implemented and successfully exploited for reasons of practical applicability of a model reduction approach for the first time in this work. For both, the offline tabulation and the online use in CFD (computational fluid dynamics) simulations whole families of optimal trajectories need to be calculated. For a specified range of the reaction progress

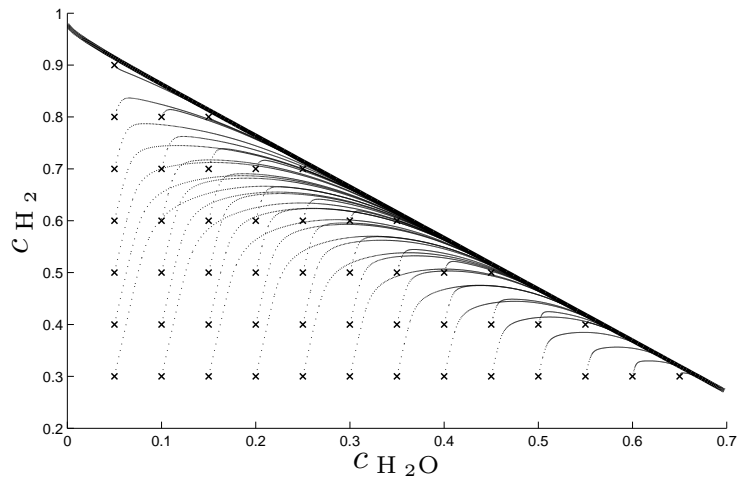

FIG. 1: Application of the initial value embedding strategy to the calculation of families of optimal trajectories illustrated for the hydrogen combustion mechanism (13): For a discrete grid of initial values of the reaction progress variables $\mathrm{H}_{2}$ and $\mathrm{H}_{2} \mathrm{O}$ (depicted as cruxes), optimal trajectories spanning a two-dimensional manifold are calculated (dotted lines).

variables, these optimal trajectories have to be calculated on a discrete grid, where neighboring grid points only slightly differ in the values of the progress variables (see Figure 1).

These ideas cannot only be used to calculate families of optimal trajectories spanning two-dimensional manifolds as demonstrated in this work, but can also easily be extended to large-scale mechanisms and the calculation of optimal trajectories spanning higher-dimensional manifolds. For most common model reduction techniques the calculation of higher-dimensional manifolds is hardly practicable due to the immense computational effort that is necessary. Using MUSCOD-II together with the initial value embedding strategy to solve the optimization problem set up in our model reduction approach results in drastically reduced computing times due to the highly accelerated convergence of the optimization problem. Therefore it should even be efficiently applicable to large-scale mechanisms for which reduced models often need to be represented by higher-dimensional manifolds.

\section{OPTIMIZATION CRITERIA}

Both success and degree of accuracy of the general trajectory based optimization approach for model reduction (1) depend on the choice of the optimization criterion $\Phi(c(t))$, which should describe the maximal relaxation of chemical forces in the underlying dynamical system. Here, we want to briefly review the criterion of minimal entropy production and subsequently develop an alternative but related choice for an optimization criterion which is motivated by fundamental considerations related to geometric interpretation of forces as curvature of trajectories.

In order to derive a thermodynamic criterion which is related to maximal relaxation of chemical forces along 
phase space trajectories, Lebiedz ${ }^{28}$ considered a generalized concept for the "distance" of a chemical system from its attractor. Under isolated conditions the attractor of a chemical system is the thermodynamic equilibrium. In Lebiedz's model reduction approach, a special trajectory (called Minimal Entropy Production Trajectory (MEPT) $)$ converging towards equilibrium is calculated such that the sum of affinities of the entropy production rates of single reaction steps is minimized $28,35,36$. The entropy production rate is closely related to the concept of chemical affinity which was first introduced by de Donder ${ }^{37}$ as the driving force of chemical reactions. For an elementary reaction step $j$ with the forward and

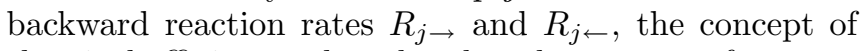
chemical affinity can be related to the concept of entropy production by the following relation ${ }^{38}$ :

$$
\frac{\mathrm{d}_{\mathrm{i}} S_{j}}{\mathrm{~d} t}=R\left(R_{j \rightarrow}-R_{j \leftarrow}\right) \ln \left(\frac{R_{j \rightarrow}}{R_{j \leftarrow}}\right),
$$

where $\mathrm{d}_{\mathrm{i}} S_{j} / \mathrm{d} t$ is the entropy production rate for reaction $j$ and $R$ is the gas constant. Entropy production rates are additive for several elementary reaction steps. Therefore the total entropy production rate (the sum of the entropy production rates of all $n$ elementary reaction steps) can be computed for an arbitrary reaction system, if kinetic data are available and a detailed elementary reaction step mechanism is known.

An intuitive justification for the minimization of the total entropy production rate in the optimization problem (1) is provided by relation (2). In partial equilibrium the entropy production rate $\mathrm{d}_{\mathrm{i}} S_{j} / \mathrm{d} t$ of a single elementary reaction step is zero, since in partial equilibrium forward and backward reaction rates are equal. This is equivalent to the thermodynamic driving force being fully relaxed, which in turn is an equivalent of the assumption of model reduction techniques based on time scale separation. There it is assumed that fast reaction modes relax into partial equilibrium or quasi-steady-states and the whole system can be satisfactorily described by the slow modes only. But unlike the methods explicitly based on time scale separation it is not necessary in the MEPT approach to actually identify and analyze the dynamical modes by e.g. numerically expensive eigenvalue decomposition and solve highly nonlinear algebraic "reduction equations". A configuration with as many elementary reaction steps as possible being close to quasi-equilibrium in a chemical sense is determined automatically by the optimization algorithm. The logarithmic ratio of forward and backward reaction rates in (2) has the meaning of a reaction affinity ${ }^{38}$. It is weighted by the absolute difference between the rates for forward and backward reactions. Thus fast processes produce more entropy than slow ones and the fast reactions have a stronger weighting factor in the optimization problem (1), which is fully natural for our purpose.

In the context of the general optimization problem (1), using entropy production as an optimization functional means

$$
\Phi(c(t))=\sum_{j=1}^{n} \frac{\mathrm{d}_{\mathrm{i}} S_{j}}{\mathrm{~d} t} .
$$

As stated above, a suitable objective functional $\Phi(c(t))$ should optimally characterize the relaxation of chemical forces. A more fundamentally rooted criterion in this context can be derived on the basis of the concept of curvature of trajectories in phase space and subsequently be combined with the entropy production ${ }^{39}$. From a physical point of view curvature is closely related to the geometric interpretation of a force.

One of the most popular examples is Einstein's general theory of relativity ${ }^{40}$ which proposes the idea that gravitational force is replaced by a "geometric picture". Einstein's general theory of relativity relates the special theory of relativity and Newton's law of universal gravitation with the insight that gravitation can be described by curvature of space-time. Space-time is treated as a 4dimensional manifold whose curvature is due to the presence of mass, energy, and momentum.

But even long before Einstein, the concept of curvature has already been related to the concept of force in physics. In 1687 Sir Isaac Newton published the laws of motion in his work "Philosophiae Naturalis Principia Mathematica". In a differential formulation Newton's second law can be stated as

$$
F=m \cdot a
$$

where $m$ is mass, $a$ is acceleration and $F$ is force. Since the acceleration $a$ is the second derivative of the state variable $x(t)$ with respect to time, $a=\ddot{x}$, and thus contains information about the curvature of $x$, in Newton's law for the first time force is directly related to curvature. In this context it is important to remark that equations of motion in classical mechanics can also be described by a variational principle, Hamilton's principle of least action. In Lagrangian mechanics, the trajectory of an object is determined such that the action (which is defined as the integral of the Lagrangian over time, where the Lagrangian is the difference of kinetic energy and potential energy) is minimal. It can be shown by using the calculus of variations, that Lagrange's equations of motion are equivalent to Hamilton's principle ${ }^{41}$.

Another well known variational principle is Fermat's principle of optics. It states in its classical form that the actual path between two points taken by a beam of light is minimal.

Our aim is to transfer the principle of "force = curvature" to the field of chemical systems and look for a corresponding variational principle formally similar to those mentioned above in a physical context.

In chemical systems dissipative forces are active. Slow and fast dynamic modes result in an anisotropic force relaxation behavior in phase space. To formally being able to describe this anisotropy for a chemical system whose dynamics are described by the ODE $\dot{c}=f(c)$, curvature 
of the trajectories in phase space is considered. The following relations hold:

$$
\begin{aligned}
\ddot{c}(t) & =\frac{\mathrm{d}^{2} c}{\mathrm{~d} t^{2}}=\frac{\mathrm{d} \dot{c}}{\mathrm{~d} t}=\frac{\mathrm{d} \dot{c}}{\mathrm{~d} c} \cdot \frac{\mathrm{d} c}{\mathrm{~d} t}=J(\dot{c}(t)) \cdot \dot{c}(t) \\
& =J(f(c(t))) \cdot f(c(t))
\end{aligned}
$$

with $J(f)$ being the Jacobian of the right hand side of the ODE $\dot{c}(t)=f(c(t))$.

Hence we may define the curvature of $c(t)$ as the vector norm

$$
\|\ddot{c}(t)\|=\|J(f(c(t))) \cdot f(c(t))\| .
$$

Transferring the fundamental geometric principle of force being equivalent to curvature mentioned above, we relate the curvature of trajectories in a kinetic model $\dot{c}=f(c)$ to the forces driving the chemical system towards equilibrium by subsequent relaxation of dynamical modes. In thermodynamic equilibrium those chemical forces become zero. In search of a criterion which characterizes maximal relaxation of chemical forces it is tempting to describe the relaxation of the system towards equilibrium by minimal remaining chemical forces, i.e. in our context by minimal total ("integrated") curvature of trajectories defined by the objective function

$$
\Phi(c(t))=\|J(f(c)) \cdot f(c)\|
$$

in the general optimization problem (1). As the curvature - here the norm of a second derivative - can be interpreted as some kind of "acceleration", the integral over the curvature in the objective function (6) is a term with the physical dimension of velocity. It correlates to the time scales present in the chemical system described by (1b). Minimizing the velocity term is related to minimizing a suitably weighted "overall" velocity of the reactions. In the optimal solution then the fast modes should be relaxed as much as possible subject to constraints comprising the system dynamics, conservation relations, and the fixation of initial values.

Interestingly, from a different point of view the objective function (6) can also be interpreted as minimizing the length of a trajectory in a suitable Riemannian metric.

For any continuously differentiable curve $\gamma(t)$ on a Riemannian manifold, the length $L$ of $\gamma$ is defined as

$$
L(\gamma)=\int_{\gamma} \sqrt{g_{\gamma(t)}(\dot{\gamma}(t), \dot{\gamma}(t))} \mathrm{d} t .
$$

with $g_{\gamma(t)}$ being a scalar product defined on the tangent space of the curve in each point. If the Riemannian metric $g_{\gamma(t)}$ is chosen as

$$
g_{\gamma(t)}(f, f):=f^{T} \underbrace{J^{T} J}_{\text {positive definite }} f=\|J f\|^{2}
$$

the "length-minimizing" objective functional equivalent to $(6)$ is now

$$
\min \int_{0}^{T} \sqrt{g_{\gamma(t)}(\dot{c}(t), \dot{c}(t))} \mathrm{d} t
$$

The solution trajectory of this problem can be interpreted as a geodesic, i.e. a curve which minimizes the length of the path between two points in a possibly curved manifold. Hence the "distance from equilibrium in a chemical sense" can be formulated here in an explicit mathematical form based on concepts from differential geometry.

To describe the distance of a chemical system from its thermodynamic equilibrium in an very general way, the Riemannian metric

$$
\hat{g}_{\gamma(t)}(f, f):=f^{T} \underbrace{J^{T} \cdot A \cdot J}_{\text {positive definite }} f=:\|J f\|_{A}^{2},
$$

can be considered, where $A$ is a positive definite matrix. As a possible choice for $A$ we propose the diagonal matrix with the entries

$$
a_{k k}=\sum_{j=1}^{n} \nu_{k j} \frac{\mathrm{d}_{\mathrm{i}} S_{j}}{\mathrm{~d} t} \quad(k=1, \ldots, m) .
$$

which represents an anisotropic "kinetic weighting" of the phase space directions by including the entropy production rate. Here $n$ is the number of reactions, $\nu_{k j}$ are the stoichiometric coefficients describing the degree to which the chemical species $k$ participates in reaction $j$, and $\mathrm{d}_{\mathrm{i}} S_{j} / \mathrm{d} t$ is the entropy production rate of reaction $j$. $a_{k k}$ is the sum of the entropy production rates of all elementary reactions in which species $k$ takes part. $A$ is positive definite since according to the Second Law of Thermodynamics $\mathrm{d}_{\mathrm{i}} S_{j} / \mathrm{d} t>0$ holds for any spontaneous process, and therefore $a_{k k}>0$ for all $k=1, \ldots, m$.

Having this, an objective function in the general problem (1) is readily formulated as

$$
\Phi(c(t))=\|J f\|_{A}
$$

which obviously includes formulation (6) for the choice $A=I_{m}$ (identity matrix).

We will demonstrate in the next section that this criterion yields highly promising results for the computation of slow attracting manifolds by help of our optimization approach.

\section{RESULTS}

To demonstrate the practical success of the proposed model reduction method we present results based on the following example mechanism for $\mathrm{H}_{2}$ combustion.

$\begin{array}{ccc}\mathrm{H}_{2} & \stackrel{k_{ \pm 1}}{=} & 2 \mathrm{H} \\ \mathrm{O}_{2} & \stackrel{k_{ \pm 2}}{=} & 2 \mathrm{O} \\ \mathrm{H}_{2} \mathrm{O} & \stackrel{k_{ \pm 3}}{=} & \mathrm{H}+\mathrm{OH} \\ \mathrm{H}_{2}+\mathrm{O} & \stackrel{k_{ \pm 4}}{=} & \mathrm{H}+\mathrm{OH} \\ \mathrm{O}_{2}+\mathrm{H} & \stackrel{k_{ \pm 5}}{=} & \mathrm{O}+\mathrm{OH} \\ \mathrm{H}_{2}+\mathrm{O} & \stackrel{k_{ \pm 6}}{=} & \mathrm{H}_{2} \mathrm{O}\end{array}$


with the rate constants

$$
\begin{array}{ll}
k_{1}=2.0, & k_{-1}=216.0 \\
k_{2}=1.0, & k_{-2}=337.5 \\
k_{3}=1.0, & k_{-3}=1400.0 \\
k_{4}=1000.0, & k_{-4}=10800.0 \\
k_{5}=1000.0, & k_{-5}=33750.0 \\
k_{6}=100.0, & k_{-6}=0.7714
\end{array}
$$

The kinetic model for the reaction mechanism is given by

$$
\begin{aligned}
& \frac{\mathrm{d} c_{\mathrm{H}_{2}}}{\mathrm{~d} t}=-k_{1} c_{\mathrm{H}_{2}}+k_{-1} c_{\mathrm{H}_{2}}^{2}-k_{4} c_{\mathrm{H}_{2}} c_{\mathrm{O}}+k_{-4} c_{\mathrm{H}} c_{\mathrm{OH}} \\
& -k_{6} c_{\mathrm{H}_{2}} c_{\mathrm{O}}+k_{-6} c_{\mathrm{H}_{2} \mathrm{O}} \\
& \frac{\mathrm{d} c_{\mathrm{H}}}{\mathrm{d} t}=2 k_{1} c_{\mathrm{H}_{2}}-2 k_{-1} c_{\mathrm{H}_{2}}^{2}+k_{3} c_{\mathrm{H}_{2} \mathrm{O}}-k_{-3} c_{\mathrm{H}} c_{\mathrm{OH}} \\
& +k_{4} c_{\mathrm{H}_{2}} c_{\mathrm{O}}-k_{-4} c_{\mathrm{H}} c_{\mathrm{OH}}-k_{5} c_{\mathrm{O}_{2}} c_{\mathrm{H}}+k_{-5} c_{\mathrm{O}} c_{\mathrm{OH}} \\
& \frac{\mathrm{d} c_{\mathrm{O}_{2}}}{\mathrm{~d} t}=-k_{2} c_{\mathrm{O}_{2}}+k_{-2} c_{\mathrm{O}}^{2}-k_{5} c_{\mathrm{H}} c_{\mathrm{O}_{2}}+k_{-5} c_{\mathrm{O}} c_{\mathrm{OH}} \\
& \frac{\mathrm{d} c_{\mathrm{O}}}{\mathrm{d} t}=2 k_{2} c_{\mathrm{O}_{2}}-2 k_{-2} c_{\mathrm{O}}^{2}-k_{4} c_{\mathrm{H}_{2}} c_{\mathrm{O}}+k_{-4} c_{\mathrm{H}} c_{\mathrm{OH}} \\
& +k_{5} c_{\mathrm{H}} c_{\mathrm{O}_{2}}-k_{-5} c_{\mathrm{O}} c_{\mathrm{OH}}-k_{6} c_{\mathrm{H}_{2}} c_{\mathrm{O}}+k_{-6} c_{\mathrm{H}_{2} \mathrm{O}} \\
& \frac{\mathrm{d} c_{\mathrm{H}_{2} \mathrm{O}}}{\mathrm{d} t}=-k_{3} c_{\mathrm{H}_{2} \mathrm{O}}+k_{-3} c_{\mathrm{H}} c_{\mathrm{OH}}+k_{6} c_{\mathrm{H}_{2}} c_{\mathrm{O}}-k_{-6} c_{\mathrm{H}_{2} \mathrm{O}} \\
& \frac{\mathrm{d} c_{\mathrm{OH}}}{\mathrm{d} t}=k_{3} c_{\mathrm{H}_{2} \mathrm{O}}-k_{-3} c_{\mathrm{H}} c_{\mathrm{OH}}+k_{4} c_{\mathrm{H}_{2}} c_{\mathrm{O}}-k_{-4} c_{\mathrm{H}} c_{\mathrm{OH}} \\
& +k_{5} c_{\mathrm{H}} c_{\mathrm{O}_{2}}-k_{-5} c_{\mathrm{O}} c_{\mathrm{OH}} \text {. }
\end{aligned}
$$

Together with the conservation relations

$$
\begin{aligned}
2 c_{\mathrm{H}_{2}}+2 c_{\mathrm{H}_{2} \mathrm{O}}+c_{\mathrm{H}}+c_{\mathrm{OH}} & =C_{1} \\
2 c_{\mathrm{O}_{2}}+c_{\mathrm{H}_{2} \mathrm{O}}+c_{\mathrm{O}}+c_{\mathrm{OH}} & =C_{2}
\end{aligned}
$$

this mechanism yields a system with four degrees of freedom.

First we investigate and extend the MEPT approach proposed by Lebiedz $z^{28,35,36}$ on the basis of the given mechanism for hydrogen combustion (13). We present results for the computation of one-dimensional slow attracting manifolds and demonstrate an extension to twodimensional manifolds, which are efficiently computed as families of MEPTs exploiting initial value embedding (see section II.) for parametric optimization in order to compute neighboring optimal trajectories.

Choosing only one reaction progress variable and fixing its initial concentration, a single trajectory with "maximally relaxed chemical forces" (here characterized by a minimal total entropy production rate) can be computed. In Figure 2 the MEPT for a fixed initial concentration of $\mathrm{H}_{2} \mathrm{O}, c_{\mathrm{H}_{2} \mathrm{O}}(0)=10^{-4}$, and the constants $C_{1}=2.0, C_{2}=1.0$ in the conservation equations is depicted as a bold black line. The equilibrium value is $\left(c_{\mathrm{H}_{2}}, c_{\mathrm{H}}, c_{\mathrm{O}_{2}}, c_{\mathrm{O}}, c_{\mathrm{H}_{2} \mathrm{O}}, c_{\mathrm{OH}}\right)=$ $(0.27,0.05,0.135,0.02,0.7,0.01)$.

As long as there is at least one degree of freedom left in the system, the problem formulation (1) permits the choice of more reaction progress variables. To illustrate how the MEPT method can be applied for model reduction to higher dimensions than one, families of MEPTs are calculated using $\mathrm{H}_{2} \mathrm{O}$ and $\mathrm{H}_{2}$ as reaction progress variables by applying the initial value embedding strategy.

First the initial concentration of $\mathrm{H}_{2}$ is varied from 0.3 to 0.95 with the initial concentration of $\mathrm{H}_{2} \mathrm{O}$ set to $10^{-4}$.

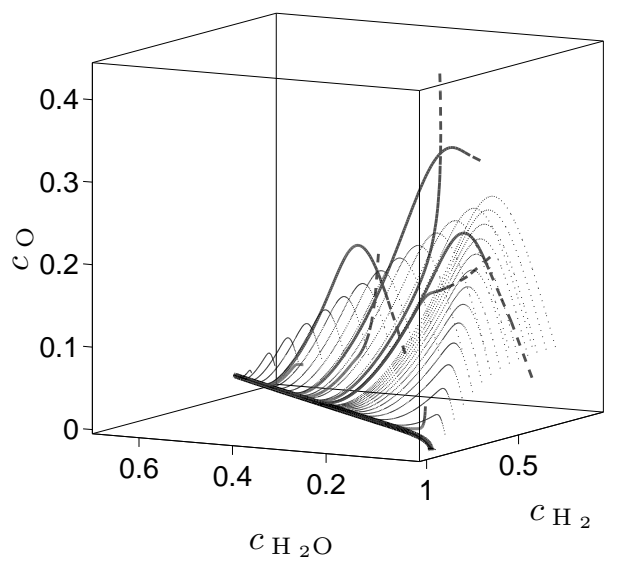

FIG. 2: Minimal Entropy Production Trajectories computed for reaction mechanism (13) as solution of problem (1) for $\Phi(c(t))=\sum_{j=1}^{n} \frac{\mathrm{d}_{i} S_{j}}{\mathrm{~d} t}$ : dotted lines represent MEPTs with $\mathrm{H}_{2} \mathrm{O}$ and $\mathrm{H}_{2}$ as reaction progress variables, which span a two-dimensional attracting manifold; the thick black line represents MEPT with only $\mathrm{H}_{2} \mathrm{O}$ as reaction progress variable; dashed lines are arbitrary trajectories bundling on the MEPT manifolds.

Then the initial concentration of $\mathrm{H}_{2}$ is set to 0.3 and the initial concentration of $\mathrm{H}_{2} \mathrm{O}$ is varied from 0.05 to 0.65 . In Figure 2 the trajectories belonging to the family of MEPTs calculated with two reaction progress variables are depicted as dotted lines. One can see that those MEPTs span a two-dimensional manifold. All of them relax to an attracting trajectory, the one-dimensional manifold (a single MEPT) calculated with just $\mathrm{H}_{2} \mathrm{O}$ as reaction progress variable and $c_{\mathrm{H}_{2} \mathrm{O}}(0)=10^{-4}$. Trajectories with arbitrary initial concentrations (plotted in Figure 2 as thick, dashed lines) all first relax to a part of the spanned two-dimensional manifold, then to the onedimensional attracting MEPT and finally to equilibrium illustrating the bundling behavior of trajectories on the computed MEPT manifolds.

When looking at the MEPTs calculated with two reaction progress variables $\mathrm{H}_{2} \mathrm{O}$ and $\mathrm{H}_{2}$ and the manifold they are spanning, one can see that the MEPTs do not start exactly on the 2-dimensional slow manifold for reaction progress variables far from their equilibrium values. They first seem to relax themselves to this manifold. This undesired behavior suggests to consider a modified optimization criterion representing relaxation of chemical forces even more accurately also under conditions far from equilibrium.

Using the criterion (12) based on a suitable "curvature=force" concept including a weighting with the entropy production rate as the objective functional, the relaxation can be completely eliminated. In Figure 3 the optimal trajectories are depicted. The fixed initial concentration of $\mathrm{H}_{2}$ is varied between 0.3 and 0.9 , and the 

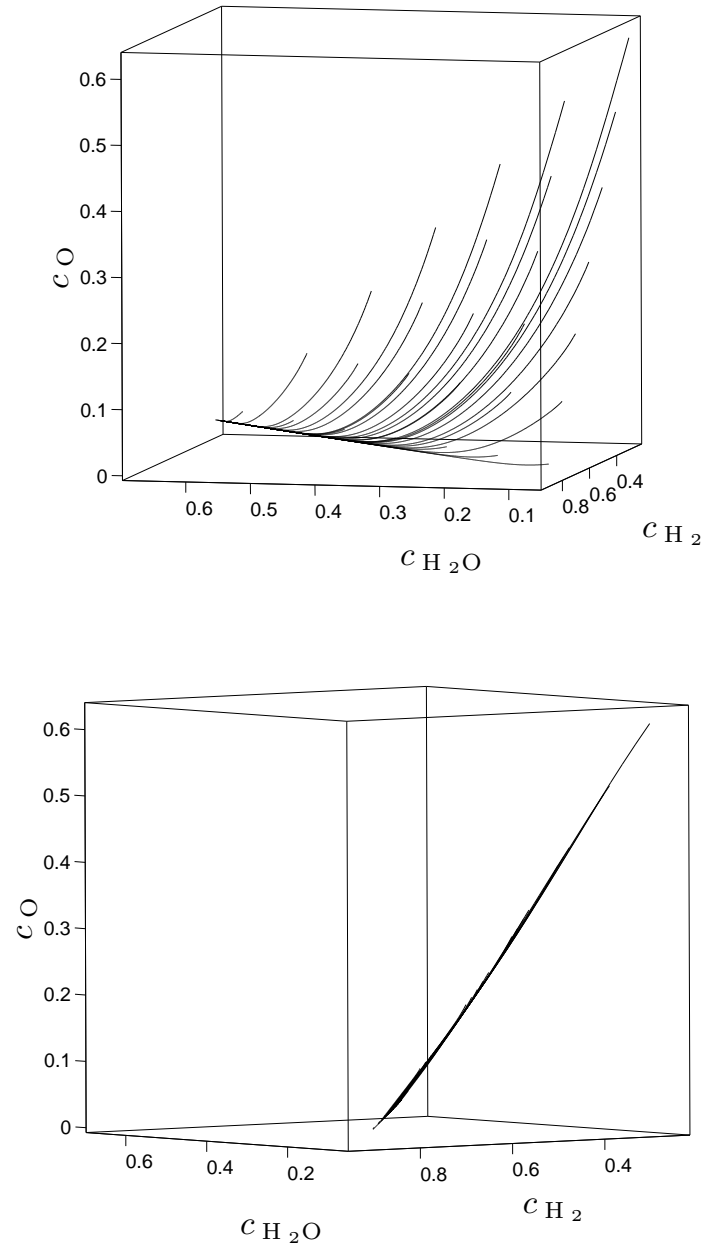

FIG. 3: Two different views of trajectories calculated as solutions of problem (1) using (12) as the objective functional.

initial concentration of $\mathrm{H}_{2} \mathrm{O}$ is varied between 0.05 and 0.65. Figure 3 shows that the optimal trajectories span exactly the two-dimensional slow attracting manifold suggesting (12) a good choice for an accurate reduction of the model mechanism (13). The computational results turn out to be largely independent of the initial values chosen for the numerical optimization. This is a highly important practical issue since it allows local species reconstruction without the requirement to compute the whole slow attracting manifold or the necessity to use continuation strategies starting near equilibrium.

\section{SUMMARY AND DISCUSSION}

We present a general framework for model reduction in chemical kinetics using an approach that is based on the optimization of trajectories. In this context, the model reduction task can be described by a variational boundary value problem related to the minimization of chemical forces, which is in principle solvable for all feasible conditions. Sophisticated numerical solution strategies exist for variational boundary problems and assure that model reduction based on the optimization of reaction trajectories presents an efficient alternative to existing model reduction approaches. The example application demonstrates promising success of the concept.

In future work, this approach will have to be adapted and tested for large-scale reaction mechanism especially using temperature dependent reaction mechanisms at low temperatures - conditions where purely timescale-based methods like the ILDM ${ }^{13}$ fail.

\section{ACKNOWLEDGEMENT}

The authors the Deutsche Forschungsgemeinschaft (German Research Foundation) for financial support, in particular in the SFB 568, the Interdisciplinary Center for Scientific Computing Heidelberg, in particular Prof. Dr. Dr. h.c. H.G. Bock for providing the MUSCODpackage and Prof. Dr. Dr. h.c. J. Warnatz for funding the project.
[1] M. S. Okino and M. L. Mavrovouniotis, Chem. Rev. 98, 391 (1998).

[2] A. N. Gorban, I. V. Karlin, and A. Y. Zinovyev, Phys. Rep. 396, 197 (2004).

[3] G. Li and H. Rabitz, Chem. Eng. Sci. 46, 95 (1991).

[4] T. Turányi, J. Math. Chem. 5, 203 (1990).

[5] H. Rabitz, M. Kramer, and D. Dacol, Annu. Rev. Phys. Chem. 34, 419 (1983)

[6] A. S. Tomlin, M. J. Pilling, T. Turanyi, J. H. Merkin, and J. Brindley, Combust. Flame 91, 107 (1992).

[7] M. Bodenstein, Z. Phys. Chem. 85, 329 (1913).

[8] D. Chapman and L. Underhill, J. Chem. Soc. Trans. 103, 496 (1913).

[9] L. Michaelis and M. L. Menten, Biochem. Z. 49, 333
(1913).

[10] M. Hadjinicolaou and D. A. Goussis, SIAM J. Sci. Comput. 20, 781 (1999).

[11] S. H. Lam and D. A. Goussis, Int. J. Chem. Kinet. 26, 461 (1994).

[12] A. N. Yannacopoulos, A. S. Tomlin, J. Brindley, J. H. Merkin, and M. J. Pilling, Physica D 83, 421 (1995).

[13] U. Maas and S. B. Pope, Combust. Flame 88, 239 (1992).

[14] U. Maas, Appl. Math. 40, 249 (1995).

[15] U. Maas, Computing and Visualization in Science 1, 69 (1998).

[16] L. Petzold and W. Zhu, AIChE Journal 45, 869 (1999).

[17] I. P. Androulakis, AIChE Journal 46, 361 (2000). 
[18] S. J. Fraser, J. Chem. Phys. 88, 4732 (1988).

[19] M. R. Roussel and S. J. Fraser, J. Chem. Phys. 93, 1072 (1990).

[20] M. R. Roussel and S. J. Fraser, J. Phys. Chem. 95, 8762 (1991).

[21] M. J. Davis and R. T. Skodje, J. Chem. Phys. 111, 859 (1999).

[22] R. T. Skodje and M. J. Davis, J. Phys. Chem. 105, 10356 (2001).

[23] M. R. Roussel and T. Tang, J. Chem. Phys. 125, 1 (2006).

[24] J. C. Keck and D. Gillespie, Combust. Flame 17, 237 (1971).

[25] D. Hamiroune, P. Bishnu, M. Metghalchi, and J. C. Keck, Combust. Theor. Model. 2, 81 (1998).

[26] Z. Ren and S. Pope, in Proceedings of the Combustion Institute (2005), vol. 30, pp. 1293-1300.

[27] Z. Ren, S. B. Pope, A. Vladimirsky, and J. M. Guckenheimer, J. Chem. Phys. 124, 114111 (2006).

[28] D. Lebiedz, J. Chem. Phys. 120, 6890 (2004).

[29] H. G. Bock and K. J. Plitt, in Proc. 9th IFAC World Congress Budapest (Pergamon Press, 1984).

[30] D. B. Leineweber, Efficient reduced SQP methods for the optimization of chemical processes described by large sparse DAE models (VDI Verlag, Düsseldorf, 1999).

[31] I. Bauer, F. Finocchi, W. J. Duschl, H.-P. Gail, and J. P. Schlöder, Astron. Astrophys. 317, 273 (1997).

[32] D. B. Leineweber, I. Bauer, H. G. Bock, and J. P. Schlöder, Comput. Chem. Eng. 27, 157 (2003).
[33] H. G. Bock, M. M. Diehl, D. B. Leineweber, and J. P. Schlöder, in Nonlinear Model Predictive Control, edited by F. Allger and A. Zheng (Birkhser, Basel, 2000), vol. 26 of Progress in Systems Theory, pp. $245-267$.

[34] M. M. Diehl, Ph.D. thesis, University of Heidelberg (2001).

[35] D. Lebiedz, V. Reinhardt, and J. Kammerer, in Model reduction and coarse-graining approaches for multi-scale phenomena, edited by A. G. et al. (Springer, Heidelberg, 2006), pp. $343-364$.

[36] D. Lebiedz, Optimal control, model- and complexityreduction of self-organized chemical and biochemical systems: A scientific computing approach, Habilitation Thesis Physical Chemistry, University of Heidelberg (2006), URL http://www.iwr.uni-heidelberg.de/groups/reaflow/ user/lebiedz/publikationen/habil_lebiedz.pdf.

[37] T. de Donder and P. van Rysselberghe, Thermodynamic Theory of Affinity: A Book of Principles (Stanford University Press, Menlo Park CA, 1936).

[38] D. Kondepudi and I. Prigogine, Modern thermodynamics (WILEY-VCH Verlag GmbH, Weinheim, 1998).

[39] M. Winckler, Master's thesis, University of Heidelberg (2007).

[40] A. Einstein, Ann. Phys. 49, 769 (1916).

[41] H. Goldstein, Classical mechanics (Addison-Wesley, Reading, Mass., 1980), 2nd ed. 